\title{
Analysis of Synthetic chemical drugs in adulterated Chinese medicines by capillary electrophoresis/ electrospray ionization mass spectrometry
}

\author{
Hsing Ling Cheng, Mei-Chun Tseng, Pei-Lun Tsai and Guor Rong Her* \\ Department of Chemistry, National Taiwan University, Taipei, Taiwan, R.O.C. \\ Received 20 April 2001; Revised 20 June 2001; Accepted 21 June 2001
}

\begin{abstract}
Sixteen synthetic chemical drugs, often found in adulterated Chinese medicines, were studied by capillary electrophoresis/UV absorbance (CE/UV) and capillary electrophoresis/electrospray ionization mass spectrometry (CE/ESI-MS). Only nine peaks were detected with CZE/UV, but on-line CZE/ MS provided clear identification for most compounds. For a real sample of a Chinese medicinal preparation, a few adulterants were identified by their migration times and protonated molecular ions. For coeluting compounds, more reliable identification was achieved by MS/MS in selected reaction monitoring mode. Micellar electrokinetic chromatography (MEKC) using sodium dodecyl sulfate (SDS) provided better separation than capillary zone electrophoresis (CZE), and, under optimal conditions, fourteen peaks were detected using UV detection. In ESI, the interference of SDS was less severe in positive ion mode than in negative ion mode. Up to $20 \mathrm{mM}$ SDS could be used in direct coupling of MEKC with ESI-MS if the mass spectrometer was operated in positive ion mode. Because of better resolution in MEKC, adulterants can be identified without the use of MS/MS. Copyright (C) 2001 John Wiley \& Sons, Ltd.
\end{abstract}

The use of Chinese medicines to maintain human health and to cure disease has a long and rich history. Because Chinese medicine is a natural based therapy, some people believe that it is more mild and less toxic than synthetic chemical drugs. However, in order to ameliorate symptoms quickly, it is not uncommon to find that synthetic chemical drugs are illegally added to traditional Chinese medicines. This practice can be harmful to health and may result in harmful side effects or drug allergies. Therefore, it is important to provide the public with reliable information about whether or not chemical drugs are present in Chinese medicines.

Conventional analytical methods in this field have involved the separation of the adulterants by thin-layer chromatography (TLC), with identification by retarding factor values and/or ultraviolet spectroscopy. These methods are in general time-consuming, labor-intensive and, more importantly, lack the specificity for high confidence in identification. Recently, methods based on highperformance liquid chromatography (HPLC) $)^{1-4}$ and capillary electrophoresis $(\mathrm{CE})^{5-9}$ have been developed for the analysis of these adulterants in Chinese medicines. In comparison with HPLC, CE is known for its superior separation efficiency and short analysis time.

Several detection techniques, such as UV, fluorescence, and electrochemical methods, have been developed for CE applications. These methods, in which migration times

*Correspondence to: G. R. Her, Department of Chemistry, National Taiwan University, Taipei, Taiwan, R.O.C.

Contract/grant sponsor: National Science Council of the Republic of China. provide the main basis for identification, generally lack the specificity required for high confidence in identification. Moreover, in the analysis of synthetic drugs in Chinese medicines, reproducible CE migration times are difficult to obtain because of the often-unavoidable modification of the capillary surface. This in turn is due to the fact that Chinese medicinal preparations are in general much more complex than synthetic drug preparations.

In Taiwan, it is illegal to add synthetic drugs to Chinese medicines, with a maximum penalty of seven years in prison. To reduce the possibility of mistrial, analytical data of the highest quality should be provided. Because of its low detection limit, abundant structural information, and, more importantly, high specificity, mass spectrometry (MS) has been considered as one of the ideal devices for chromatographic detection. ${ }^{10-12}$ Thus, on-line coupling of CE with mass spectrometry is very attractive for the direct identification of the dubious compounds in complex mixtures. In recent years, CE/MS methods based on atmospheric pressure ionization (API) have received considerable attention for the analysis of nonvolatile and thermally labile compounds. ${ }^{13-30}$ In CE/MS, identification is based not only on the migration times, but also on the molecular ions and structurally characteristic fragment ions.

Sixteen chemical drugs (Fig. 1), often found in adulterated Chinese medicines, were studied by capillary electrophoresis/electrospray ionization mass spectrometry. The separations achieved with capillary zone electrophoresis (CZE) and micellar electrokinetic chromatography (MEKC) were compared. The feasibility of on-line coupling of MEKC with ESI- 

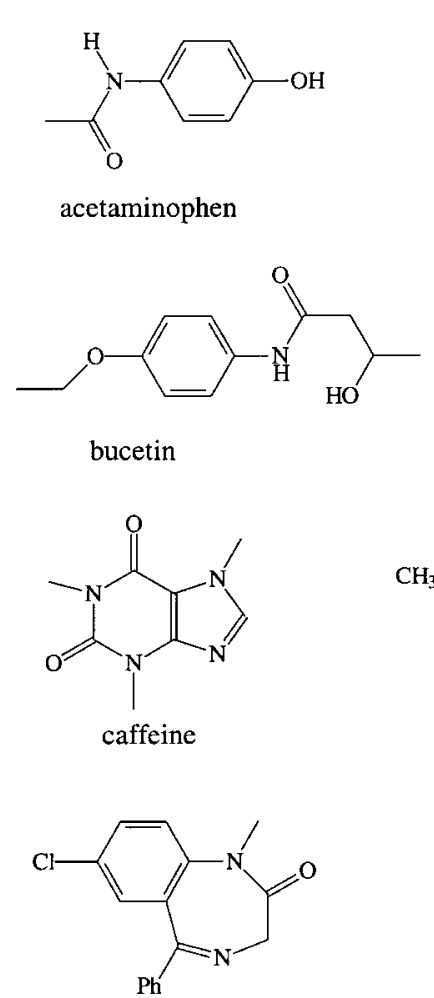

diazepam<smiles>CCOc1ccccc1C(N)=O</smiles>

ethoxybenzamide

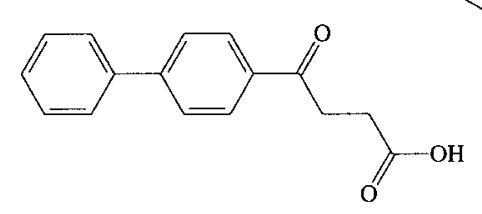

fenbufen<smiles>O=C(O)c1ccccc1Nc1cccc(C(Cl)(Cl)Cl)c1</smiles><smiles>COc1ccc2c(c1)C(CC(=O)O)=C(C)C2C(=O)c1ccc(Cl)cc1</smiles>

indomethacine<smiles>CC(C(=O)O)c1cccc(C(=O)c2ccccc2)c1</smiles>

ketoprofen<smiles>Cc1cccc(Nc2ccccc2C(=O)O)c1C</smiles><smiles>O=C(O)c1cccnc1Nc1cccc(C(F)(F)F)c1</smiles>

niflumic acid<smiles>CCCCC1C(=O)N(c2ccccc2)N(c2ccc(O)cc2)C1=O</smiles>

oxyphenbutazone<smiles>CCCCC1C(=O)N(c2ccccc2)N(c2ccccc2)C1=O</smiles><smiles>CC12C=CC(=O)C=C1CCC1C2CCC2(C)C1CC[C@@]2(O)C(=O)CO</smiles>

prednisolone<smiles>NC(=O)c1ccccc1O</smiles><smiles>CC1=C(CC(=O)O)c2cc(F)ccc2/C1=C\c1ccc(S(C)=O)cc1</smiles>

Figure 1. Structures of the sixteen chemical drugs.

MS is discussed as well as the potential and the limitations of CZE/ESI-MS and MEKC/ESI-MS in the analysis of chemical drugs in Chinese medicines.

\section{EXPERIMENTAL}

\section{Reagents and materials}

All chemical drugs, acetaminophen, bucetin, caffeine, diazepam, ethoxybenzamide, fenbufen, flufenamic acid, indomethacine, ketoprofen, mefenamic acid, niflumic acid, oxyphenbutazone, phenylbutazone, prednisolone, salicylamide and sulindac, were obtained from the Laboratories of Foods and Drugs, Dept. of Health, Taipei, Taiwan. Methanol, ammonium acetate and formic acid were of chromatographic grade from J. T. Baker (Phillipsburg, NJ, USA). Ethanol was of HPLC-grade, purchased from Merck
(Darmstadt, Germany). Sodium dodecyl sulfate (SDS) was purchased from Sigma (St. Louis, MO, USA). Deionized (18 $\mathrm{M} \Omega$ ) water (Milli-Q water system, Millipore Inc., Bedford, MA, USA) was used in the preparation of the buffer solution. Chemical drugs were dissolved in ethanol. The concentration of each was $100 \mathrm{ppm}$ for direct-infusion ESI-MS analysis, and 1000 ppm for CE/UV, CE/ESI-MS and CE/ESIMS/MS analysis.

\section{Preparation of sample extract}

Chinese medicine pills were obtained from National Laboratories of Foods and Drugs, Dept. of Health, Taipei, Taiwan. Each sample was pounded into powder. A sample of $100 \mathrm{mg}$ was dissolved in $5 \mathrm{~mL}$ ethanol, extracted for $30 \mathrm{~min}$ by ultrasound, and centrifuged for $1 \mathrm{~min}$ to remove 


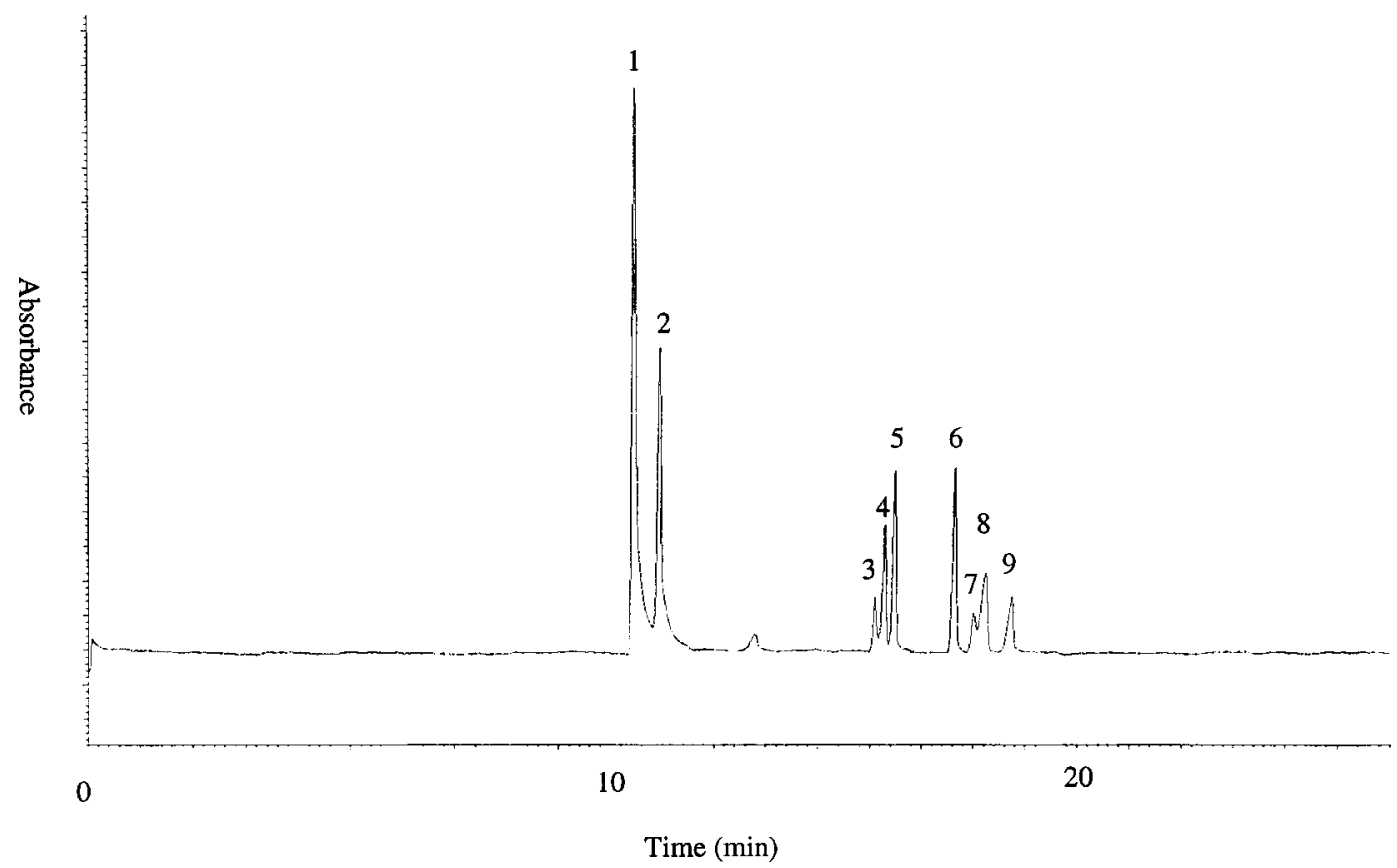

Figure 2. CZE/UV electrophorogram of the sixteen chemical drugs. A $90-\mathrm{cm}$ (80-cm effective length) fusedsilica capillary tubing was used with $40 \mathrm{mM}$ ammonium acetate at $\mathrm{pH} 9$ as running buffer. The potential was $+20 \mathrm{kV}$ and the detection wavelength was $254 \mathrm{~nm}$. Samples were injected hydrodynamically (15 mbar for $10 \mathrm{~s}$ ). Peaks were assigned as (1) salicylamide, ethoxybenzamide, caffeine, bucetin, diazepam, and prednisolone (2) acetaminophen (3) phenylbutazone, and oxyphenbutazone (4) sulindac (5) indomethacine (6) fenbufen (7) niflumic acid (8) ketoprofen (9) flufenamic acid, and mefenamic acid.

the undissolved sample from the solution. The extract was filtered with a $0.45-\mu \mathrm{m}$ filter and then concentrated to $50 \mu \mathrm{L}$.

\section{CE system}

The CE system was constructed in-house and has been described elsewhere. ${ }^{31} \mathrm{CE}$ columns were fused-silica capillary (Polymicro Technologies, Phoenix, AZ, USA) $50 \mu$ m i.d., $375 \mu \mathrm{m}$ o.d. and $90 \mathrm{~cm}$ length $(80 \mathrm{~cm}$ to the UV detector). A small area of the polyimide coating was burned off to form a window for UV detection. On-column detection was performed with a UV detector (UV-C Rainin, Emeryville, CA, USA) operated at $254 \mathrm{~nm}$. The MEKC running buffers were $20 \mathrm{mM}$ SDS and $40 \mathrm{mM}$ ammonium acetate, $\mathrm{pH}$ 9. The capillary was washed with $1 \mathrm{M} \mathrm{NaOH}$, followed by $0.1 \mathrm{M}$ $\mathrm{NaOH}$, water, and running buffer. The capillary was equilibrated with running buffer for 20 min before each run.

\section{Mass spectrometry}

A Finnigan LCQ quadrupole ion trap mass spectrometer equipped with an electrospray ionization source (Finnigan MAT; San Jose, CA, USA) was used. The spray voltage was set to $+4 \mathrm{kV}$ and the heated capillary was set to $200^{\circ} \mathrm{C}$. Solutions of chemical drugs (100 ppm) were infused with a syringe pump at a flow rate of $5 \mu \mathrm{L} / \mathrm{min}$. The CE/MS interface utilizes a triaxial flow arrangement whereby $\mathrm{CE}$ eluent is mixed with a suitable make-up sheath liquid at the tip and nebulized by nitrogen gas. CE columns were $80 \mathrm{~cm}$ long. The high voltage applied to the buffer reservoir was $24 \mathrm{kV}$. With $+4 \mathrm{kV}$ ESI voltage applied at the outlet of the separation column, the potential difference during the analysis was about $20 \mathrm{kV}$ for CE/ESI-MS analysis. The sheath liquid consisted of water, methanol, and formic acid (30:70:0.2, v/v/v) and was delivered at a flow rate of $3 \mu \mathrm{L} /$ min by syringe pump. The mass spectrometer was operated in positive ion mode, and data were collected in full scan mode for CE/ESI-MS analysis and selected reaction monitoring (SRM) mode for CE/ESI-MS/MS analysis. In MS/MS analysis, voltage for the collision energy (peak-to-peak amplitude of the resonance excitation) ranged from 0.6 to $1 \mathrm{~V}$. To avoid space charge effects, the number of ions stored in the trap was regulated by the automatic gain control, which was set at $5 \times 10^{7}$ ions for full scan mode, and $1.2 \times 10^{7}$ for SRM mode. Helium was used as damping gas at a pressure of $10^{-3}$ Torr. The maximum ion collection time was $200 \mathrm{~ms}$ for each step and 3 scans were averaged for each spectrum. The LCQ mass spectrometer was operated at unit resolution.

\section{RESULTS AND DISCUSSION}

CZE/UV and CZE/MS analysis of synthetic drugs Because of the ease of on-line coupling with ESI-MS, CZE was first chosen to separate the sixteen chemical drugs. Under the optimal condition $(40 \mathrm{mM}$ ammonium acetate at $\mathrm{pH}$ 9), nine peaks were detected by UV (Fig. 2). Coinjection with authentic standards suggested that six analytes: salicylamide, ethoxybenzamide, caffeine, bucetin, diazepam, and prednisolone, coeluted and also migrated at a velocity equal to the EOF (peak 1). In addition to this first coeluting peak, phenylbutazone coeluted with oxyphenbutazone (peak 3) and flufenamic acid coeluted with mefenamic acid (peak 9). In the analysis of synthetic drugs from the crude 


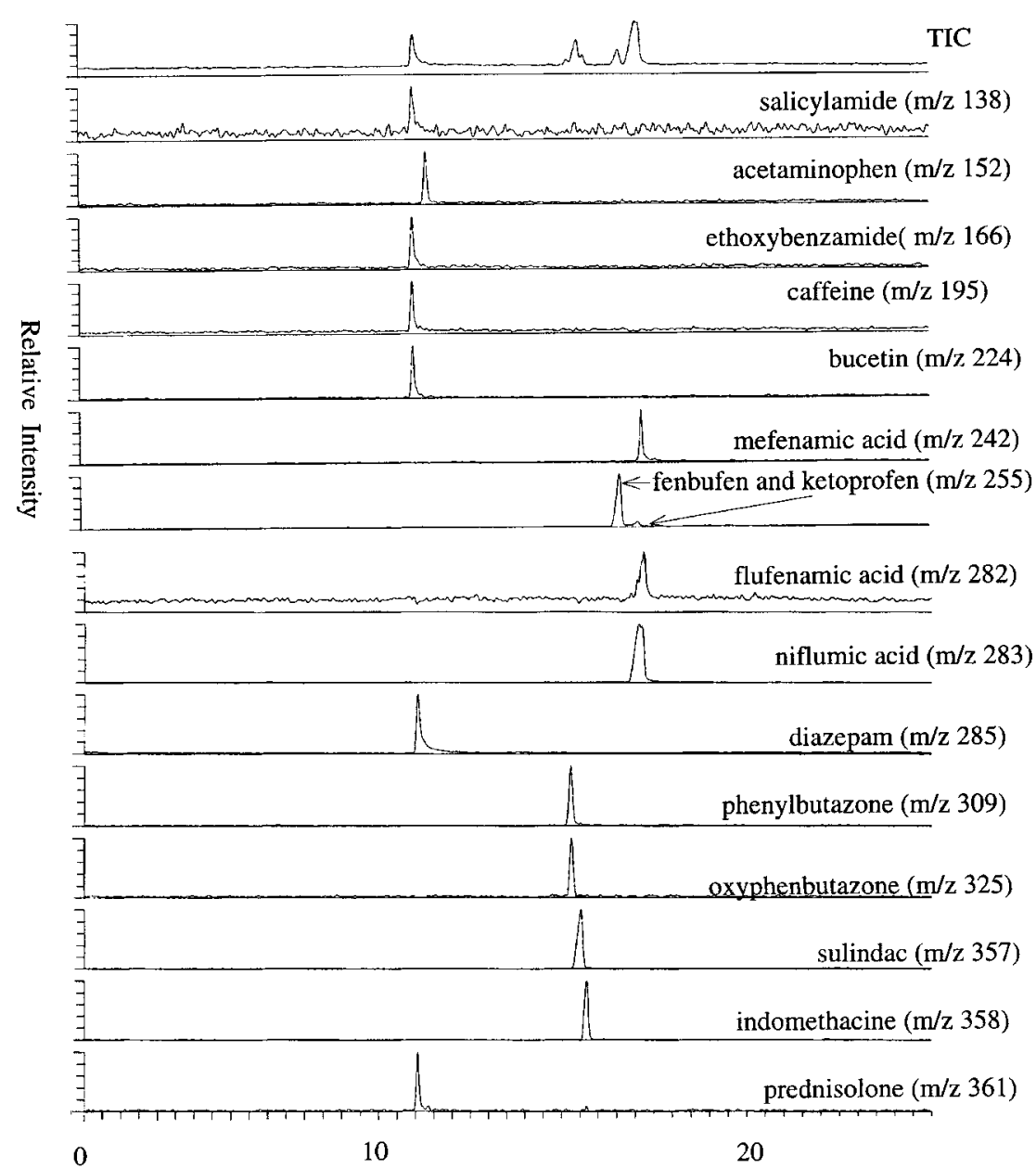

Time (min)

Figure 3. CZE/ESI-MS electrophorograms of the sixteen chemical drugs. Sheath liquid composition was methanol/water (70:30) containing $0.2 \%$ formic acid. The potential applied to the buffer reservoir was $+24 \mathrm{kV}$, and the ESI voltage was set at $+4 \mathrm{kV}$. Data were collected in full scan mode over the range $\mathrm{m} / \mathrm{z} 100-400$.

extract of Chinese medicinal preparations, it was found that the reproducibility of migration time was poorer than in the analysis of pure standards. This is most likely due to capillary surface modification by the complex matrix in the crude extract. The poor reproducibility in migration time makes identification solely based on migration time not reliable.

Because of its very high specificity, mass spectrometry would be a better detector for CE than UV. Furthermore, with MS as the detector, baseline resolution of components may not be needed for a clear identification. Accordingly, CZE was coupled directly with ESI-MS using a sheath liquid interface. The CZE separation was carried out under basic conditions ( $\mathrm{pH}$ 9). To perform positive ion ESI-MS, analytes should preferably be converted to protonated molecules $\left([\mathrm{M}+\mathrm{H}]^{+}\right)$before entering the electrospray needle. This was achieved with the help of an acidic sheath liquid (methanol/ water/formic acid, 70:30:0.2, v/v/v). The mass electrophorograms of the sixteen drugs are shown in Fig. 3.

Several real samples were studied by this CZE/MS method. The results of these analyses suggested that, in cases with inadequate CE resolution, MS/MS would be a better choice for the identification of chemical drugs in Chinese medicines. For example, four compounds were detected in one Chinese medicinal preparation, but, of these four compounds, three compounds coeluted. In addition, the fourth compound had a migration time very similar to that of the first three. In this case, the detection specificity based on migration time and/or molecular weight might not be adequate for a clear identification. To improve the confidence of compound identification, MS/MS was used. The major fragment ions resulting from collision-induced dissociation (CID) are listed in Table 1. For better sensitivity, only one transition was monitored for each analyte. As shown in Fig. 4, four synthetic drugs were identified in this real sample. Three compounds were eluted at $11.2 \mathrm{~min}$. The $m / z 195 \rightarrow 138$ transition corresponds to the loss of $\mathrm{CH}_{3} \mathrm{NCO}$ from the molecular ion of caffeine. The $m / z 166 \rightarrow 149$ transition corresponds to the loss of $\mathrm{NH}_{3}$ from the molecular ion of ethoxybenzamide and the $\mathrm{m} / \mathrm{z} 361 \rightarrow 343$ transition corresponds to the loss of $\mathrm{H}_{2} \mathrm{O}$ from the molecular ion of prednisolone. The $\mathrm{m} / \mathrm{z} 152 \rightarrow 110$ transition at $11.6 \mathrm{~min}$ 
Table 1. Protonated molecular ions and their major CID fragment ions for the sixteen chemical drugs

\begin{tabular}{lcl}
\hline Chemical drug & $\begin{array}{c}\text { Protonated molecule } \\
\text { ion }(\mathrm{m} / \mathrm{z})\end{array}$ & Major fragment ions \\
\hline Acetaminophen & 152 & 110 \\
Bucetin & 224 & 206138 \\
Caffeine & 195 & 138 \\
Diazepam & 285 & 257228224182154 \\
Ethoxybenzamide & 166 & 149 \\
Fenbufen & 255 & 237 \\
Flufenamic acid & 282 & 264 \\
Indomethacine & 358 & 174139 \\
Ketoprofen & 255 & 209 \\
Mefenamic acid & 242 & 224 \\
Niflumic acid & 283 & 265 \\
Oxyphenbutazon & 325 & 297232204188 \\
Phenylbutazone & 309 & 211188160 \\
Prednisolone & 361 & 343325307 \\
Salicylamide & 138 & 121 \\
Sulindac & 357 & 340297248 \\
\hline
\end{tabular}

corresponds to the loss of $\mathrm{C}_{2} \mathrm{H}_{2} \mathrm{O}$ from the molecular ion of acetaminophen.

\section{MEKC/UV and MEKC/MS analysis of synthetic drugs}

MEKC is known for its excellent resolving power in the separation of charged as well as neutral compounds. ${ }^{32}$ To improve the separation of the sixteen synthetic drugs, MEKC was then studied as the separation method.

MEKC was not selected initially because on-line coupling of MEKC with ESI-MS is generally considered much more difficult than the coupling of CZE with ESI-MS. Surfactants such as SDS are known to reduce ionization efficiency, and lead to ion source contamination. ${ }^{20}$ Several approaches including $\mathrm{APCI}^{21,22}$ coupled capillary, ${ }^{23}$ high molecular weight surfactant, ${ }^{30}$ partial-filling $\mathrm{MEKC}_{,}^{24-27}$ and anodically migrating micelles, ${ }^{28}$ have been proposed to alleviate this problem. Recently, it has been reported that MEKC can be coupled directly with ESI in the positive selected ion monitoring (SIM) mode. ${ }^{29}$

Direct coupling of MEKC with ESI-MS was investigated in this study because it required the least amount of technical manipulation. To investigate the feasibility of analyzing adulterants in Chinese medicines by direct coupling of MEKC with ESI-MS, the effect of SDS on ESI efficiency was studied using infusion experiments. The results of negative ion ESI showed that, even at a SDS concentration of $10 \mathrm{mM}$, which is only slightly higher than the critical micelle concentration value $(8.2 \mathrm{mM})$, the signals of analytes were seriously suppressed. In positive ion ESI-MS mode, the interference was less severe. The effect of SDS on analyte signals as a function of SDS concentration is shown in Fig. 5.

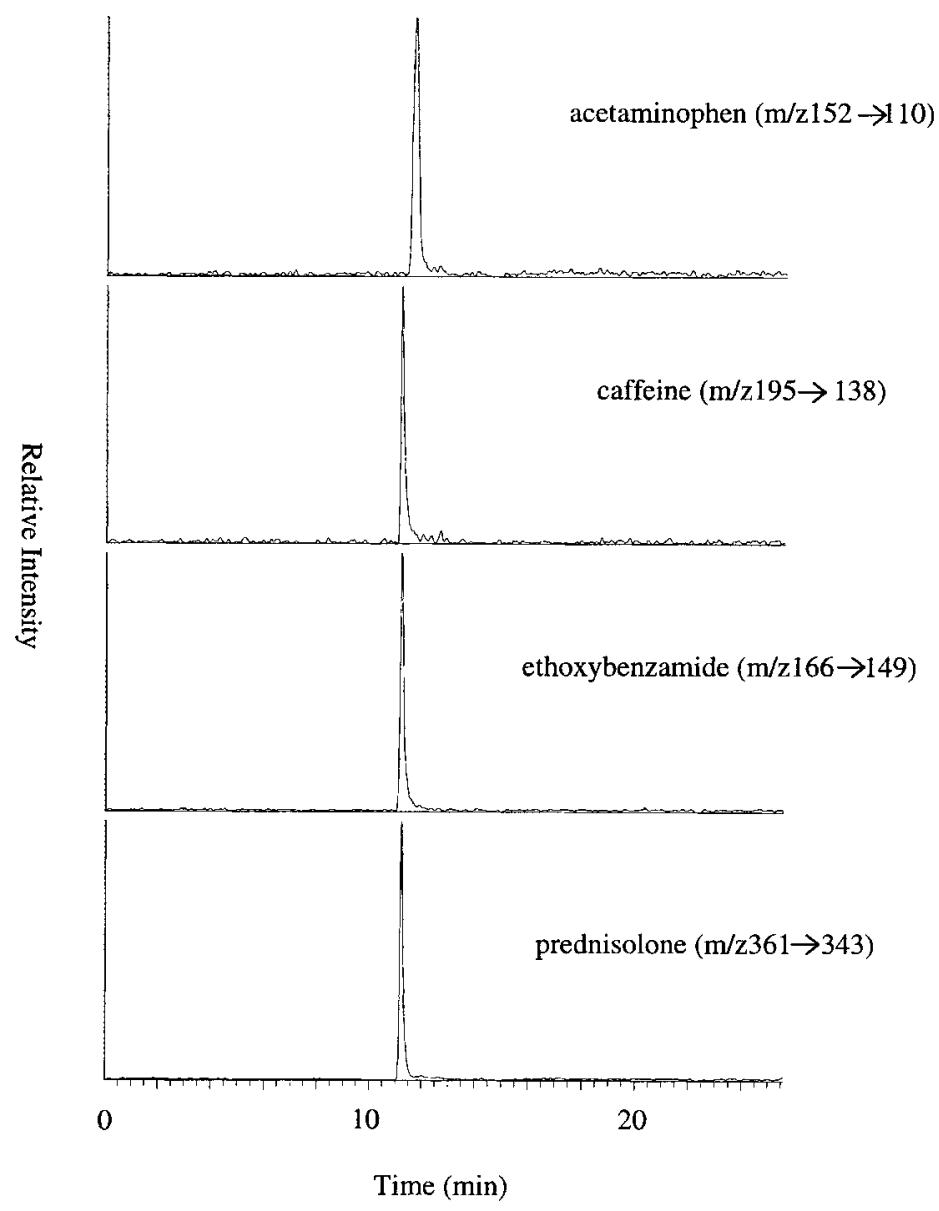

Figure 4. CZE/ESI-MS/MS electrophorograms of a real sample. The CZE and MS conditions were the same as in Fig. 3. Data were collected in SRM mode. 


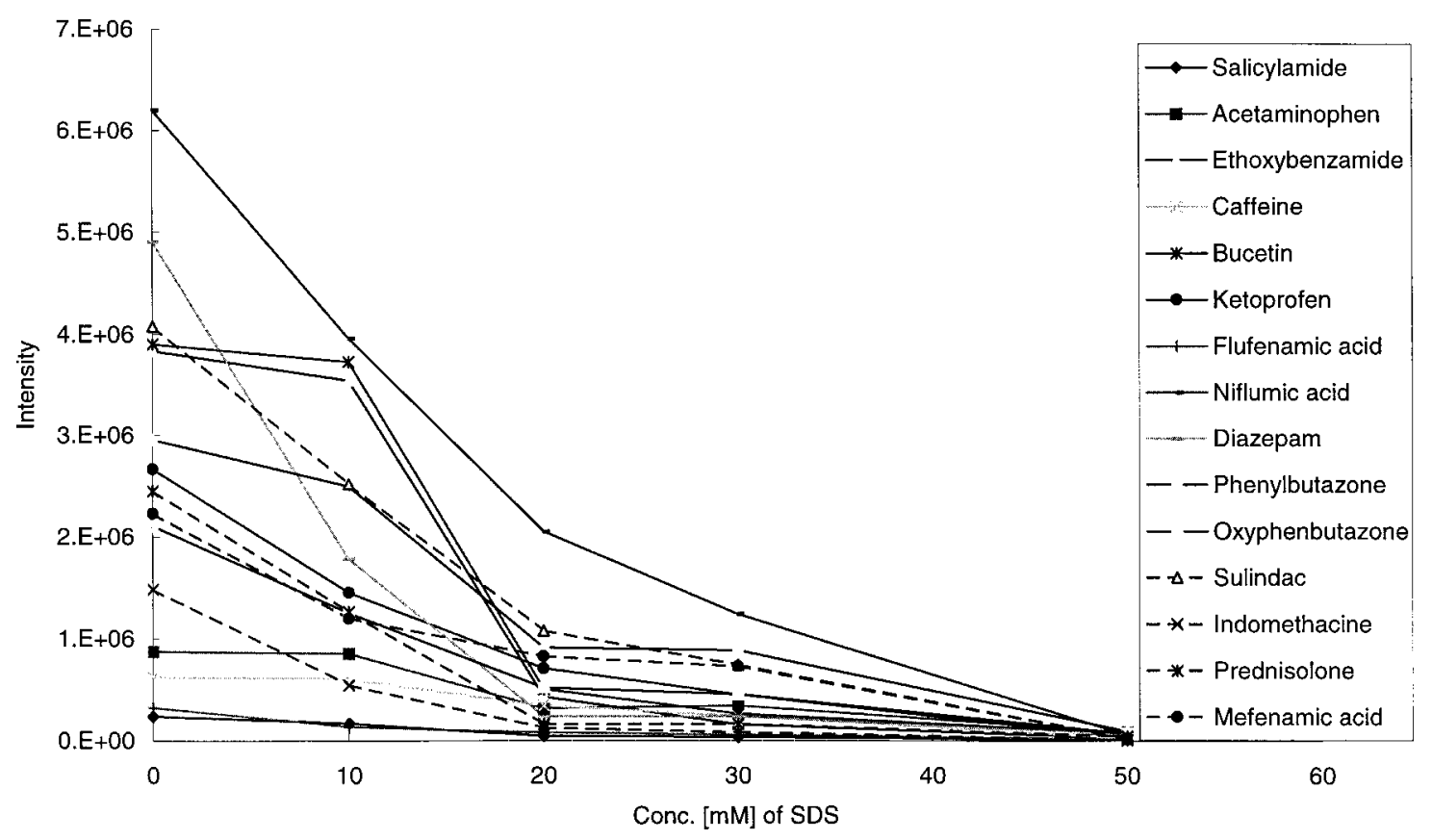

Figure 5. Effect of SDS on analyte signals as a function of SDS concentration. The concentration of each analyte was $100 \mathrm{ppm}$.

The infusion experiment showed that, with SDS concentrations greater than $20 \mathrm{mM}$, the signals were reduced to less than $30 \%$ for most analytes in comparision with those observed with no SDS. Accordingly, the sixteen analytes were separated at SDS concentrations less than $20 \mathrm{mM}$. The results showed that $20 \mathrm{mM}$ gave much better separation than
$10 \mathrm{mM}$, and the separation (Fig. 6) appeared to be significantly better than that obtained using CZE. Fourteen instead of nine peaks were observed with UV detection. Coinjection with authentic standards suggested that ethoxybenzamide coeluted with salicylamide, and mefenamic acid coeluted with flufenamic acid. Among the fourteen peaks, many of

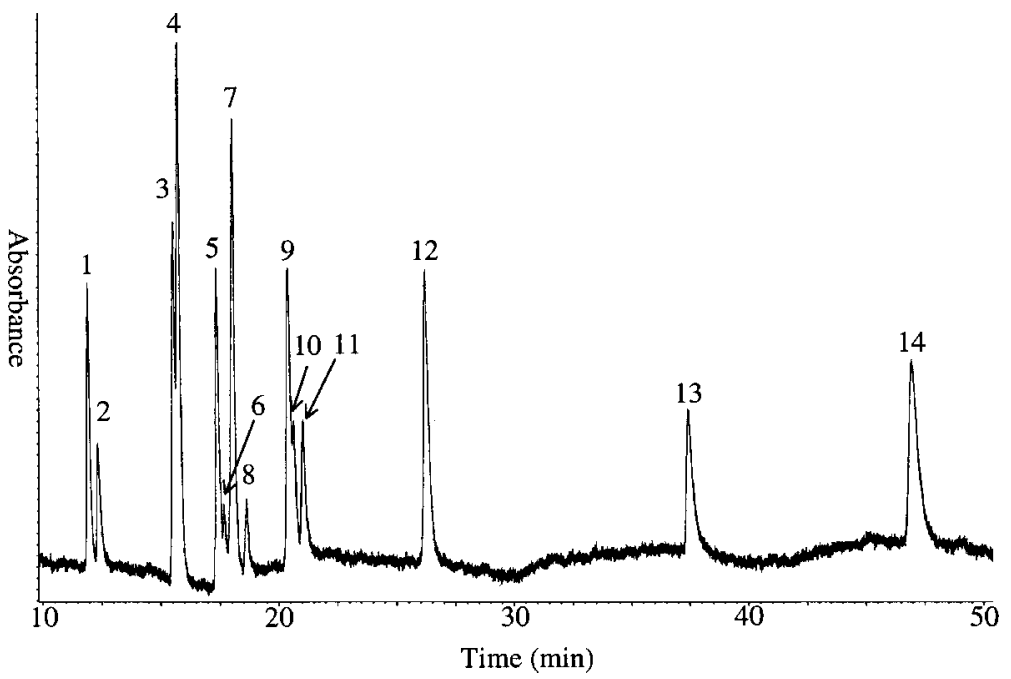

Figure 6. MEKC/UV electrophorogram of the sixteen chemical drugs. A 90$\mathrm{cm}$ (80-cm effective length) fused-silica capillary tubing was used with $20 \mathrm{mM}$ SDS and $40 \mathrm{mM}$ ammonium acetate at $\mathrm{pH} 9$ as running buffer. The potential was $+20 \mathrm{kV}$ and the detection wavelength was $254 \mathrm{~nm}$. Samples were injected hydrodynamically ( $15 \mathrm{mbar}$ for $10 \mathrm{~s}$ ). Peaks were assigned as (1) acetaminophen (2) caffeine (3) oxyphenbutazone (4) phenylbutazone (5) bucetin (6) ethoxybenzamide, salicylamide (7) fenbufen (8) niflumic acid (9) sulindac (10) mefenamic acid, flufenamic acid (11) ketoprofen (12) indomethacine (13) prednisolone (14) diazepam. 


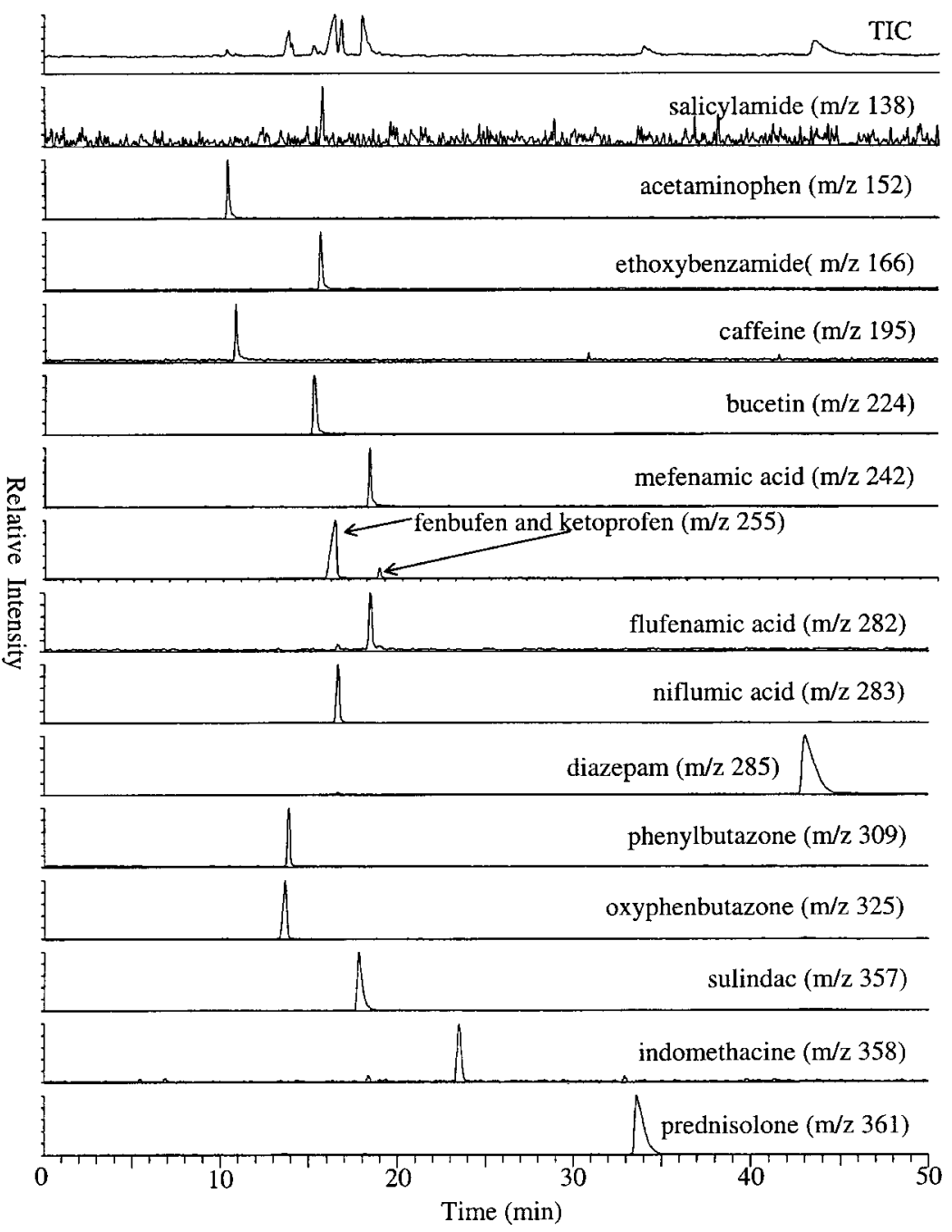

Figure 7. MEKC/ESI-MS electrophorograms of the sixteen chemical drugs. Sheath liquid composition was methanol/water (70:30) containing $0.2 \%$ formic acid. The potential applied to the buffer reservoir was $+24 \mathrm{kV}$ and ESI voltage was set at $+4 \mathrm{kV}$. Data were collected in full scan mode over the range $\mathrm{m} / \mathrm{z} 100-400$.

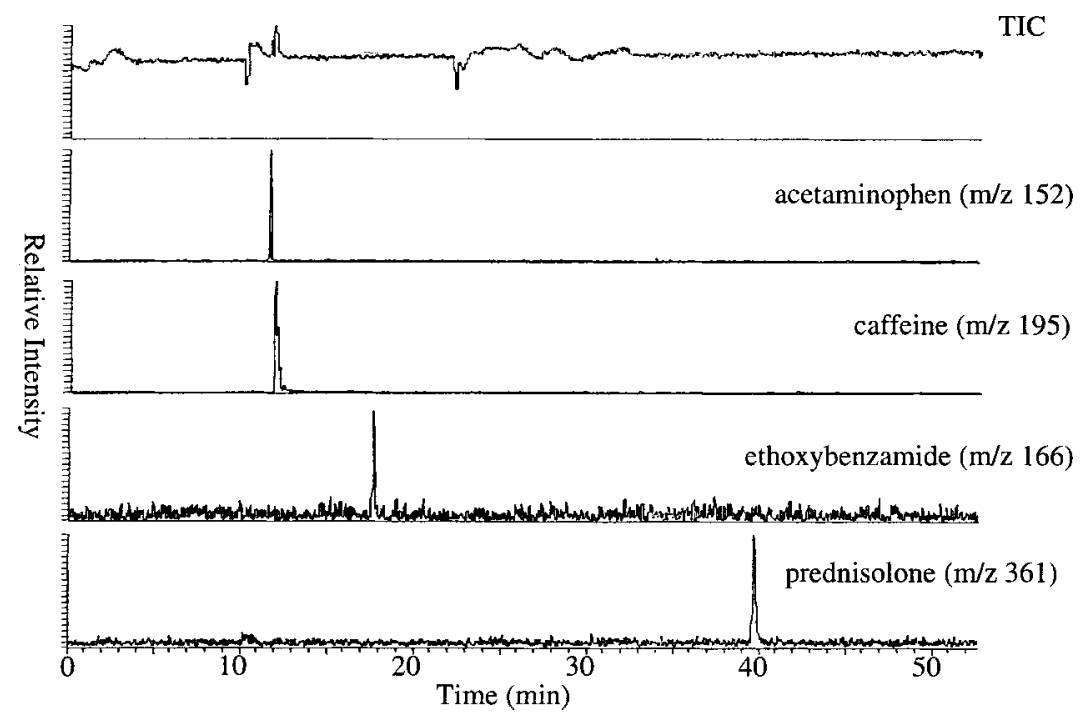

Figure 8. MEKC/ESI-MS electrophorograms of a real sample. The MEKC and MS conditions were the same as those used to obtain Fig. 7. Data were collected in full scan mode over the range $\mathrm{m} / \mathrm{z} 100-400$. 
them were only partially resolved. In order to achieve a clear identification of the presence of synthetic drugs in adulterated Chinese medicines, MEKC was coupled with ESI-MS.

Direct coupling of MEKC with ESI-MS appeared to be more difficult than the coupling of CZE with ESI-MS; the signals were not as stable as in CZE/MS, and the electrical continuity was relatively difficult to maintain. Despite these difficulties, we were able to analyze these synthetic drugs, as shown in Fig. 7. The isobaric compounds ketoprofen and fenbufen result in the appearance of two peaks in the chromatogram for $[\mathrm{M}+\mathrm{H}]^{+}$ions at $m / z$ 255. Several real samples have been analyzed by this MEKC/ESI-MS method. The MEKC/ESI-MS reconstructed mass chromatograms of a real sample are shown in Fig. 8. The sample used in this MEKC/MS analysis is the same as that used for Fig. 4. Unlike the CZE/MS approach (Fig. 4), MS/MS was not necessary in this MEKC/MS study because of better MEKC separation.

\section{CONCLUSIONS}

Because of the lack of adequate resolution in CZE, MS/MS may be needed for the identification of adulterants in Chinese medicines. MEKC provided better separation than CZE in the analysis of the sixteen chemical drugs investigated here. However, it is more difficult to couple MEKC with ESI-MS. Currently, we are investigating the merits and pitfalls of these two approaches in quantitative analysis of the sixteen drugs. It is known that the signal of an analyte may be affected by the presence of other electrolytes. This may create some problems in the quantitative analysis of coeluting adulterants, especially under conditions in which isotopically labeled internal standards are not available.

\section{Acknowledgements}

We thank the National Science Council of the Republic of China for financial support.

\section{REFERENCES}

1. Misaki T, Sagawa L, Ojima T, Kakizawa S, Oshima T, Yoshizawa H. Chem. Pharm. Bull. 1982; 30: 354.

2. Luo G, Wang Y, Zhou G, Yu Y. J. Liq. Chromatogr. 1990; 13: 3825.

3. Tsai TH, Hong CY, Chen CF. Yaowu Shipin Fenxi 1997; 5: 303.

4. Ku CC, Chung YS, Ku H. Yaowu Shipin Fenxi 1994; 2: 289.

5. Sheu SJ, Lu CF. J. High Rssolut. Chromatogr. 1996; 19: 409.

6. Ku YR, Tsai MJ, Wen KC. Yaowu Shipin Fenxi 1995; 3: 185.

7. Sheu SJ. J. Chin. Chem. Soc. 1996; 54: 55.

8. Liebich HM, Lehmann R, Stefano CD, Haring HU, Kim JH, Kim KR. J. Chromatogr. A 1998; 795: 388.

9. Shen Q, Fang Z. Fenxi Shiyanshi 1998; 17: 1.

10. Wolfender JL, Hostettmann K. J. Chromatogr. 1990; 647: 191.

11. Aramendia MA, Garcia I, Lafont F, Marinas JM. J. Chromatogr. A 1995; 707: 327.

12. Song Y, Her GR, Wen KC. Yaowu Shipin Fenxi 1997; 5: 295.

13. Zeleny. J. Phys. Rev. 1917; 10: 1.

14. Dole M, Hines RL, Mack LL, Mobley RC, Ferguson LD, Alice MB. Macromolecules 1968; 1: 96.

15. Yamashita M, Fenn JB. J. Phys. Chem. 1984; 88: 4451.

16. Yamashita M, Fenn JB. J. Phys. Chem. 1984; 88: 4671.

17. Olivares JA, Nguyen NT, Yonker CR, Smith RD. Anal. Chem. 1987; 59: 1230.

18. Udseth HR, Barinaga CJ, Smith RD. Anal. Chem. 1988; 60: 1948.

19. Lee ED, Henion W, Muck JD, Covey TR. J. Chromatogr. 1988; 645: 313.

20. Kriger MS, Cook KD. Anal. Chem. 1995; 67: 358.

21. Takada Y, Sakairi M, Koizumi H. Anal. Chem. 1995; 67: 1474.

22. Takada Y, Sakairi M, Koizumi H. Rapid Commun. Mass Spectrom. 1995; 9: 488.

23. Lamoree MH, Tjaden UR, Greef JVD. J. Chromatogr. A 1995; 712: 219.

24. Nelson WM, Lee CS. Anal. Chem. 1996; 68: 3265.

25. Nelson WM, Tang Q, Harrata AK, Lee CS. J. Chromatogr. A 1996; 749: 219.

26. Wiedmer SK, Jussila M, Riekkola ML. Electrophoresis 1998; 19: 1711.

27. Muijselaar PG, Otsuka K, Terabe S. J. Chromatogr. A 1998; 802: 3.

28. Yang L, Harrata AK, Lee CS. Anal. Chem. 1997; 69: 1820.

29. Tanaka Y, Kishimoto Y, Otsuka K, Terabe S. J. Chromatogr. A 1998; 817: 49.

30. Ozaki H, Itou N, Terabe S, Takada Y, Sakairi M, Koizumi H. J. Chromatogr. A 1995; 716: 69.

31. Tsai CY, Her GR. J. Chromatogr. A 1996; 743: 315.

32. Terabe S, Otsuka K, Ichikawa K, Tsuchikya A, Ando T Anal. Chem. 1984; 56: 111. 